\title{
Frontières
}

\section{Fantastique cadavre}

\section{Patrick Bergeron}

Volume 23, numéro 2, printemps 2011

Enquêtes sur le cadavre : 2. Fantastique

URI : https://id.erudit.org/iderudit/1007582ar

DOI : https://doi.org/10.7202/1007582ar

Aller au sommaire du numéro

Éditeur(s)

Université du Québec à Montréal

ISSN

1916-0976 (numérique)

Découvrir la revue

Citer ce document

Bergeron, P. (2011). Fantastique cadavre. Frontières, 23(2), 5-6.

https://doi.org/10.7202/1007582ar

Ce document est protégé par la loi sur le droit d'auteur. L'utilisation des services d'Érudit (y compris la reproduction) est assujettie à sa politique d'utilisation que vous pouvez consulter en ligne.

https://apropos.erudit.org/fr/usagers/politique-dutilisation/
Cet article est diffusé et préservé par Érudit.

Érudit est un consortium interuniversitaire sans but lucratif composé de l’Université de Montréal, l'Université Laval et l'Université du Québec à Montréal. Il a pour mission la promotion et la valorisation de la recherche. https://www.erudit.org/fr/ 


\section{FANTASTIQUE CADAVRE}

\section{Patrick Bergeron,}

directeur du numéro.

Depuis l'aube des temps, l'être humain prend soin de ses morts. Selon les anthropologues, la mise en place de rituels funéraires fait même partie des plus anciens indices d'un passage à la civilisation. Depuis un âge tout aussi reculé, l'humain s'est mis à élaborer un ensemble très étendu de croyances, superstitions, peurs, fables et discours de tous genres quant au devenir posthume non seulement de l'âme, mais aussi du cadavre.

Et pourtant, comme il est difficile, à la vue de la dépouille d'un être cher, de se résigner à l'idée que son corps ne s'animera plus jamais! Le problème peut aussi être pris à l'inverse: combien de cauchemars ont été inspirés à l'homme par la crainte d'un retour à la vie des défunts! Des ombres que rencontre Ulysse au chant XI de L'Odyssée aux lugubres inferi du monde d'Harry Potter, des goules des folklores arabe et perse aux morts-vivants de George Romero, le cadavre a, de tout temps, occupé une grande place dans l'imaginaire fantastique. En dépit des efforts que déploie toute société pour départager fermement l'espace des vivants de celui des morts, l'imagination a toujours permis à l'humain de transgresser les frontières. Une prodigieuse cohorte de vampires, fantômes, momies, zombies et autres revenants est apparue dans ce cadre. Du lot, Dracula et Frankenstein sont assurément les plus célèbres, car de simples créatures de papier qu'ils étaient à la base, les personnages inventés par Bram Stoker et Mary Shelley sont devenus de véritables archétypes modernes. Pour amplifier la délicieuse terreur qu'ils nous inspirent, une vaste symbolique macabre s'est développée, composée de décors inquiétants (cimetières, maisons hantées, cryptes, oubliettes, ruines...), de malédictions, de livres empoisonnés, d'ossements... Devant l'importance qu'occupent les morts dans l'imaginaire fantastique, il nous a paru indiqué d'y consacrer le second volet de notre enquête sur le cadavre.

Un premier regroupement de textes est consacré aux créatures réchappées d'outre-tombe. À partir du roman classique de Mary Shelley et d'une nouvelle de l'écrivain croate Ksaver Šandor Gjalski, Isabelle Percebois examine l'épouvante issue des morgues et des salles de dissection du XIX ${ }^{\mathrm{e}}$ siècle, période d'intense expérimentation scientifique sur les cadavres. Fanny Robles étudie la momie victorienne, depuis sa consécration comme objet de musée et «fétiche impérial» dans la société britannique du XIX ${ }^{\mathrm{e}}$ siècle jusqu'à ses plus récents avatars hollywoodiens. S'inspirant du récent regain d'intérêt pour la figure du vampire grâce notamment aux séries de romans Twilight et True Blood ainsi qu'à leurs adaptations pour le petit et le grand écran, Deerie Sariols Persson fournit des pistes de réflexion sur l'évolution de ce mythe moderne depuis le XVIII ${ }^{\mathrm{e}}$ siècle. 
La figure abominable à laquelle je me suis intéressé n'est peutêtre pas un revenant; elle a pourtant partie liée avec les morts, dans un registre qui combine, de plus, cannibalisme et érotisme. Appartenant à l'univers des terreurs infantiles, tout comme le marchand de sable et autres croquemitaines, l'ogre occupe assurément une place de choix parmi les vilains les moins recommandables de l'imaginaire fantastique. J'ai tiré prétexte de la réapparition de l'ogre dans la fiction contemporaine pour adultes afin d'examiner une figure complémentaire qui, étonnamment, a moins retenu l'attention des chercheurs : l'ogresse. Trois romans de Paul Morand, Gabrielle Wittkop et Dynah Psyché m'ont servi à m'interroger sur l'esthétique de l'abjection et la thématique du mal infligé aux enfants.

Dans les deux textes qu'il signe ici, Luc Breton a suivi, si l'on peut dire, le cadavre à la trace : d'abord, à l'intérieur d'une microenquête menée auprès d'un complexe funéraire de Québec ; ensuite, à l'occasion d'une exposition sur les pratiques funéraires présentée au Musée québécois de culture populaire à TroisRivières. Breton nous amène à voir la thanatopraxie et tout ce qui s'y rapporte d'un tout nouvel œil.
D'autres contributions à notre enquête abordent des sujets liés à la spiritualité. Sébastien Baud examine les rapports au cadavre de la société awajún, en se concentrant sur l'usage de plantes psychotropes comme mode favorisant la rencontre avec l'âme des guerriers du passé. Nathalie Dietschy se tourne vers l'appropriation, par certains artistes contemporains, de l'iconographie du Christ mort afin d'en donner des visions personnelles et profanes.

Enfin, nous avons réuni des contributions éclairant divers aspects de la culture populaire. Francis Daoust et Jean-Guy Nadeau tentent d'expliquer la position privilégiée qu'occupent la mort et le cadavre au sein de la musique heavy metal, alors qu'Isabelle-Rachel Casta analyse la valeur centrale impartie aux cadavres, morgues et médecins-légistes dans la littérature, le cinéma et les téléséries d'aujourd'hui. La mort peut-elle encore être considérée comme un tabou?, se demande Édith Roy. L'auteure s'appuie sur l'exposition Memento Mori / Bone Again pour réfléchir aux enjeux actuels de la représentation et de la représentabilité du cadavre dans la production artistique.

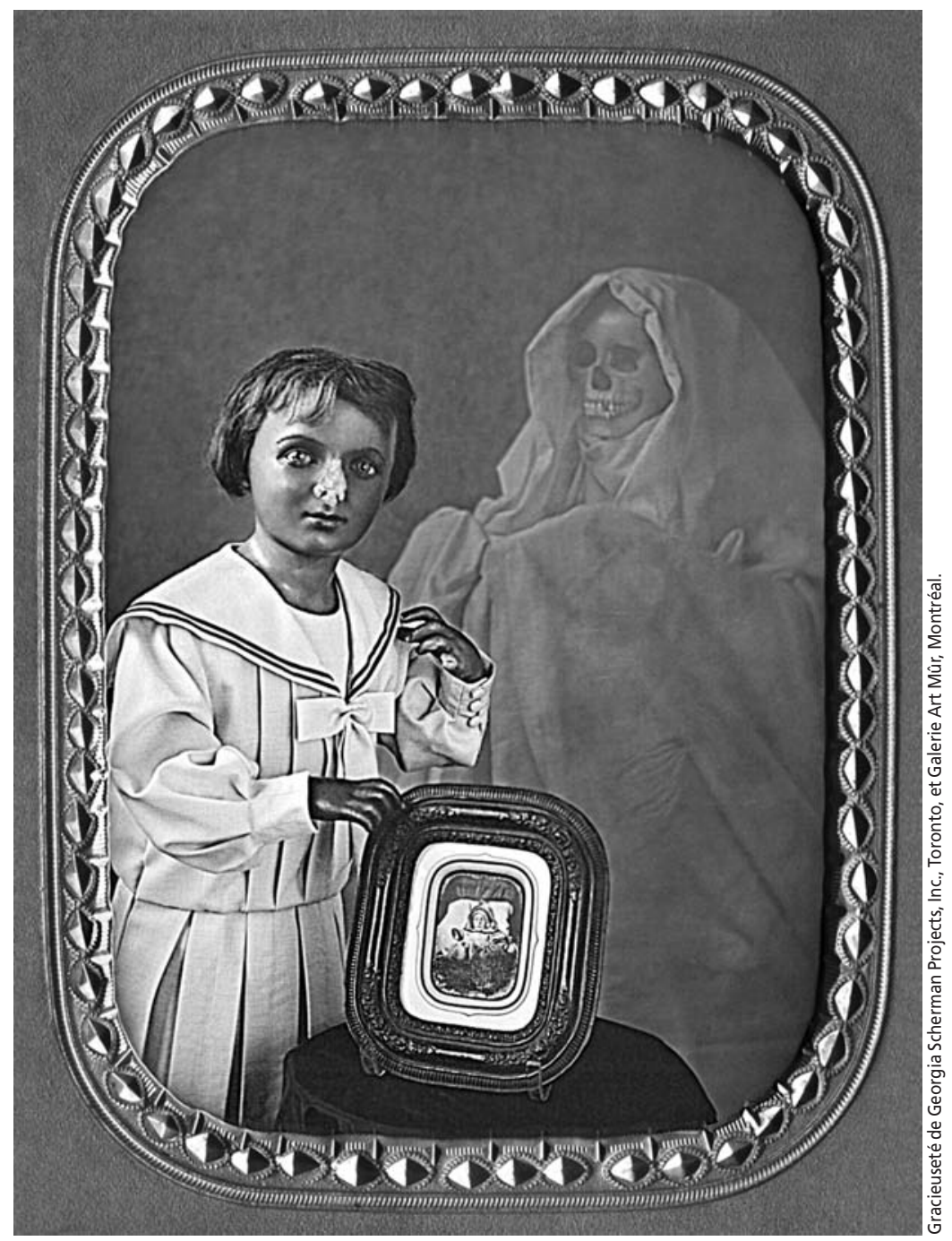

Spring Hurbut, The Visitation of the Mannequin, 2003 (collaboration avec Mike Robinson), daguerréotype et boîtier, $13 \times 10 \mathrm{~cm}$. 